\title{
Advanced mid-IR Solid-State Laser Developments
}

\author{
Jirong $\mathrm{Yu}$ \\ NASA Langley Research Center \\ MS 468, Hampton, VA 23681-2199, USA
}

\begin{abstract}
This paper reviews the state-of-the-art 2-micron solid-state laser developments. A world record one-Joule-per-pulse energy laser system and an advanced thermal management with fully conductive cooled laser technique are discussed
\end{abstract}

\section{INTRODUCTION}

The development of a space-qualifiable 2-micron laser is beneficial to both the NASA Exploration and Earth science technology goals. Coherent-detection lidar system at 2micron wavelength measures horizontal wind velocity with a high measurement precision. The same lidar system can also measure atmospheric $\mathrm{CO}_{2}$ concentration profiles, and therefore density. Ground based 2- $\mu \mathrm{m}$ coherent Doppler wind lidars and $\mathrm{CO}_{2}$ Differential Absorption Lidars (DIALs) by using 2-micron laser transmitters at output energy of 100 $\mathrm{mJ}$ level have been successfully demonstrated [1].

Recently, significant advancements in the laser development have been made under the NASA Laser Risk Reduction Program. One Joule per pulse energy was achieved by a Master-Oscillator-Power-Amplifier (MOPA) system. To our knowledge, it is the highest recorded energy at this wavelength. Fully conductive cooled 2-micron laser was demonstrated by the use of heat pipe technique. It enhanced the laser thermal management, and virtually eliminated the running coolant to increase the overall system efficiency and reliability. All these technical development efforts push forward the readiness of the space-borne coherent Doppler lidar.

\section{ONE-JOULE DOUBLE-PULSED HO:TM:LuLF MASTER- OSCILLATOR-POWER-AMPLIFIER (MOPA)}

The space borne coherent Doppler wind lidar and $\mathrm{CO}_{2}$ Differential Absorption Lidar (DIAL) requires high laser pulse energy, narrow line width and good beam quality [2]. To demonstrate a multi-Joule 2- $\mu \mathrm{m}$ laser, a MasterOscillator-Power-Amplifier (MOPA) laser system is required. The schematic of the experimental setup for the MOPA laser system is shown in Figure 1.

The laser oscillator consists of two curved high reflectors, one flat reflector and one flat output coupler and was symmetrically configured for a stable operation. Two curved high reflectors made two Gaussian beam waists inside the cavity. The laser crystal rod and a Brewsterangle-cut fused-silica acousto-optical Q-switch were located at these two Gaussian beam waists respectively. The oscillator output is first amplified by a preamplifier. The first amplifier was set at a double-pass configuration. Double-pass or multiple-pass amplifier helps to extract more pulse energy from the amplifiers to increase the output pulse energy and to improve the efficiency of the amplifiers. The second amplifier was setup at a single-pass configuration due to the limitation of the damage threshold of the laser crystal rod. The curved high reflectors are used to direct the laser beam in to the amplifiers for the compensation of the beam divergence.

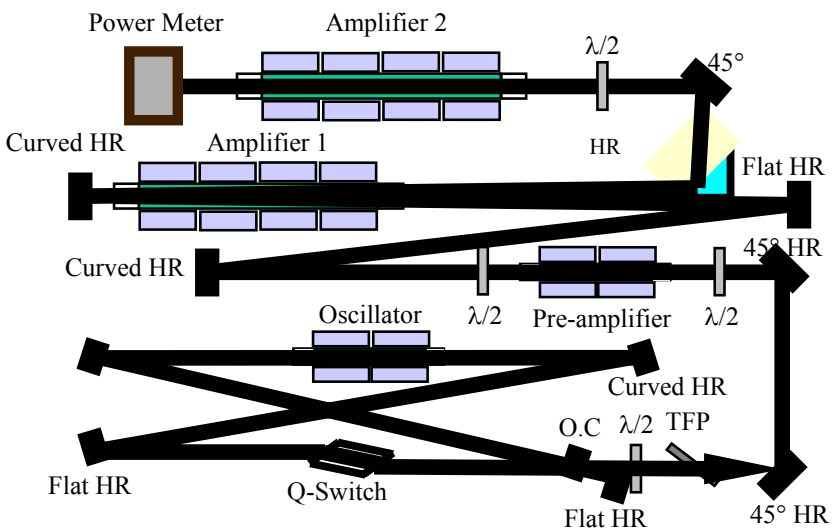

Fig. 1. Schematic of the experimental setup for one Joule MasterOscillator-Power-Amplifier (MOPA) laser system

The designed laser MOPA system could operate at normal mode, single pulse Q-switched mode, and double pulse Q-switched mode. The double Q-switch operation takes advantage of the long life time of the Ho up-level and the Tm and Ho energy sharing. This feature is ideal for the DIAL measurement where two pulses at different wavelength are needed. The total output pulse energy was measured as a function of total optical pump pulse energy for three operation modes, and they are shown in Figure 2.

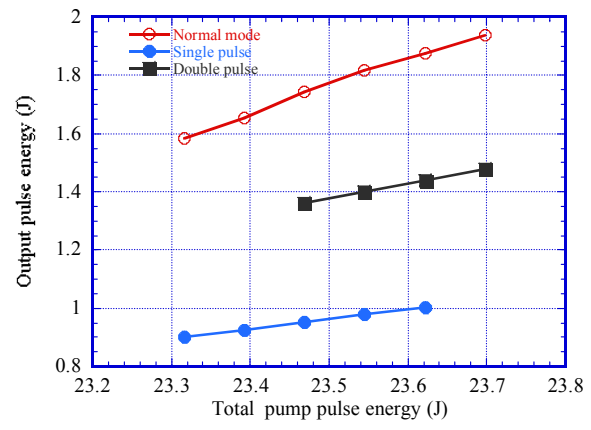

Fig. 2. MOPA ooutput pulse energy as a function of the total optical pump pulse energy 
The maximum output pulse energy at the total optical pump pulse energy of about $23.7 \mathrm{~J}$ was $1.92 \mathrm{~J}, 1.5 \mathrm{~J}$, and $1.0 \mathrm{~J}$ for the normal mode, double Q-switch and single Q-switched pulse, respectively. It is the first time that a solid-state 2micron laser breaks the Joule level barrier.

\section{CONDUCTIVE COOLED 2-MICRON LASER DEVELOPMENT}

In most of the $2-\mu \mathrm{m}$ laser developments, simple traditional method of circulating refrigerated water to remove the heat satisfies the cooling requirements. However, it is not cost effective or practical if the laser system is to be used in an environment where there are power and weight constraints and the use of water is not acceptable. The primary motivations for designing a total conductively cooled laser are to demonstrate the technologies leading to space laser application, and to enhance the overall wall plug efficiency of the laser by passively cooling the laser. This system is designed with heat pipes capable of removing heat both from the diode lasers and the rod using capillary action and transferring the heat to a radiator. In space operation, a radiator can dissipate the heat by merely facing deep space.

Fig. 3 depicts the totally conductive cooled laser head module. The six pump diode lasers are placed $10 \mathrm{~mm}$ away from the laser crystal. They are arranged $120^{\circ}$ apart around the rod. A systematic illumination analysis by using ray tracing was performed to determine the optimum design with maximum coupling efficiency for various coupling methods such as lens duct, cylindrical lenses, cylindrical reflector, conic parabolic concentrator and plane mirror reflector. The result of the study showed that plane mirrors can form a light guide superior than any of the other schemes examined. Up to $97 \%$ of the light is delivered to the rod.

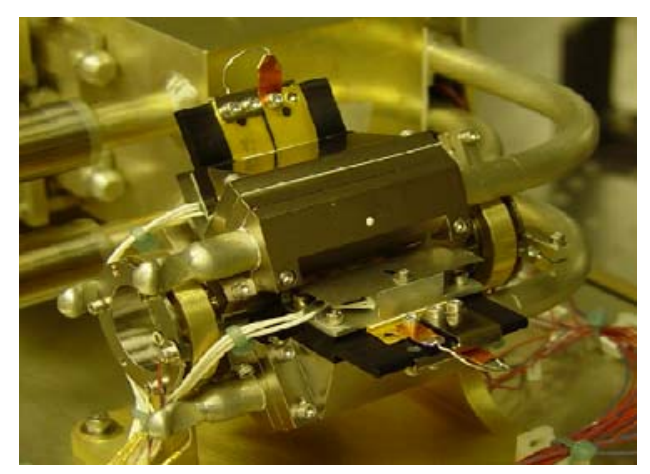

Fig. 3. Totally conductively cooled laser head assembly

Given the poor thermal conductivity of the laser material and the limited contact surface area, it is extremely technically challenging to effectively remove heat from the laser rod. The Ho:Tm:LuLF crystal, which is $4 \mathrm{~mm}$ in diameter, has only $1.52 \mathrm{~cm}^{2}$ accessible for heat removal and the rest is used for optical pumping. The laser rod is clamped between Thermkon heat sink using Nusil as interface. In addition to high thermal conductivity of thermkon, its thermal expansion coefficient is close to that of the laser crystal which helps minimize the additional mechanical stress.

The laser rod sub-assembly and the laser diodes subassembly are connected to a heat pipe. The heat generated from both laser diodes and laser rod was dissipated through the same heat pipe to a heat sink, from which the heat is removed. For space application, this heat sink can be eliminated. The heat load determines the size of the heat pipes while the minimum temperature determines the type of fluid used. Three heat pipes are used with total heat transfer capability of $150 \mathrm{~W}$ at $-50^{\circ} \mathrm{C}$ at 0.001 -inch adverse elevation. The heat pipes are attached to a chiller block that is cooled down to $-34^{\circ} \mathrm{C}$. The laser head is sealed in a box made of aluminum. The box is purged with dry nitrogen to avoid condensation.

Maximum energy of $230 \mathrm{~mJ}$ at normal mode and $107 \mathrm{~mJ}$ at Q-switch mode is achieved for an oscillator with slope efficiency of $14.5 \%$ and $6.4 \%$, respectively. Nearly half of the normal mode energy is converted to Q-switched energy. The laser is operated very well for the repetition rate up to $10 \mathrm{~Hz}$ at various heat loads.

\section{CONCLUSION}

In summary, we have developed a Tm:Ho:LuLF laser Master-Oscillator-Power-Amplifier (MOPA) system with energy exceeding one Joule level. The total efficiency of the laser MOPA system is $4.2 \%$ and $6.3 \%$ for the single pulse and double pulse, respectively.

A totally conductive cooled 2-micron laser has been successfully demonstrated. This marks a significant milestone for developing a space-qualifiable laser. Despite the thermal gradient that was created in the Ho:Tm:LuLF crystal due to the cooling method and geometry, near diffraction limited beam and up to $107 \mathrm{~mJ}$ of Q-switched output with a pulse length of $135 \mathrm{~ns}$ was obtained. This endeavor is deemed beneficial in a system where the power consumption and weight of a system is to be kept at a minimum without compromising performance.

\section{ACKOWLEDGMENT}

This work was supported by Laser Risk Reduction Program, funded by NASA Explorations and Science Directorates. The author gratefully acknowledges the contributions from Yingxin Bai, Songsheng Chen, Grady Koch, Tony Melak, Ed. Modlin, Muligeta Petros, Bo Trieu, and the program support from Upendra Singh and Michael Kavaya.

\section{REFERENCES}

[1] Grady J. Koch, B. W. Barnes, M. Petros, J. Y. Beyon, F. Amzajerdian, J.Yu, R. E. Davis, S. Ismail, S. Vay, M. J. Kavaya and U. N. Singh, "Coherent differential absorption lidar measurements of CO2", Appl. Opts, Vol. 43, No. 26, 5092-5099 (2004)

[2] J. Yu, U. N. Singh, N. P. Barnes and P. Petros, "125-mJ diode pumped injection seeded Ho:Tm:YLF laser”, Opt. Lett. 23, 780-782, 1998 